\title{
Novel MCM Interconnection Analysis Using Capacitive Charge Generation (CCG)
}

\author{
Edward I. Cole Jr., Kenneth A. Peterson, and Daniel L. Barton \\ Failure Analysis Department \\ Electronics Quality/Reliability Center \\ Sandia National Laboratories \\ Albuquerque, New Mexico 87185-1081

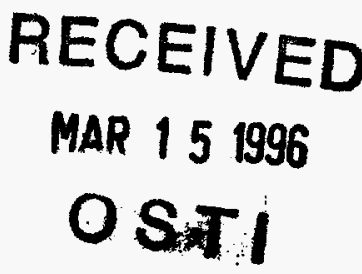

Abstract: A new SEM technique, Capacitive Charge Generation (CCG), has been developed to rapidly image MCM interconnection continuity. The new technique uses low primary electron beam energies $(<2.0 \mathrm{keV})$, very high beam currents $(>100 \mathrm{nA})$, and fast electron beam scan rates ( $>5$ frames/second) to probe buried conductors in MCMs. For these conditions, new surface charging effects have been observed that enable examination of conductors under thick insulating layers. CCG has been applied to conductors covered by over $90 \mu \mathrm{m}$ of polymer dielectric. The physics of CCG signal generation and applications for MCM failure analysis are described.

\section{INTRODUCTION}

Multi-Chip Modules (MCMs) present new challenges and opportunities for failure analysis, not only in the investigation of defects on integrated circuits (ICs) but also the MCM interconnections between the ICs and other system components [1]. Examination of MCM conductor failures is complicated by the thick passivation and interlevel dielectric layers, which can be more than an order of magnitude thicker than comparable layers in ICs. Scanning electron microscopy (SEM) techniques such as Resistive Contrast Imaging (RCI) [2] and Charge-Induced Voltage Alteration (CIVA) [3] are useful for localizing open circuits in unbiased conductors and biased interconnections respectively, but are limited to structures that are within the penetration depth of the primary electron beam. At $40 \mathrm{keV}$ (a very high SEM beam energy), the penetration depth is about $10 \mu \mathrm{m}$ for $\mathrm{SiO}_{2}$ and $\mathrm{Si}_{3} \mathrm{~N}$; a depth which is insufficient for many MCM technologies.

We have overcome the dielectric thickness limitations of conventional MCM interconnection testing by developing a new SEM imaging technique, Capacitive Charge Generation (CCG). In CCG, a charge is capacitively induced on buried conductors through thick dielectric layers. The development of CCG is a result of recent experiments on insulator surfaces using low primary electron beam energies, high beam currents, and rapid beam scan rates. At high beam currents the dielectric surface potential has an electron flux and scan rate dependence and varies as the beam scans over the surface. This ac charging effect was first reported as a method to produce CIVA images at low primary beam energies through dielectric layers (low energy CIVA or LECIVA) [4]. LECIVA is performed at beam energies that result in a positive surface potential under normal, low beam current conditions. CCG can also be performed at higher beam energies above the "cross-over" point, where the surface potential is negative under normal beam current conditions [5]. This previously undocumented surface potential dependence on scan rate and beam current for beam energies above the "cross-over" point produces a larger CCG signal than that generated at lower energies. The increase in CCG signal improves the image quality of samples with thicker dielectric layers.

An additional method for producing capacitively induced charge with energies above the "cross-over" point involves changing the primary electron beam energy as the sample is scanned. The surface equilibrium voltage will vary in proportion to a primary beam energy change.

In this work we describe the electron beam and sample interaction physics proposed to explain CCG and present several examples of its application to MCM failure analysis. Operational guidelines and limitations are also described.

\section{PHYSICS OF CCG}

CCG is analogous to the CIVA technique, in that the sample itself is the detector. Unlike CIVA, there is no signal amplification from transistor saturation. CCG images are produced directly from the current induced in a buried conductor by the polarization of the overlying dielectric.

\section{CCG at Low Primary Electron Beam Energies}

Insulator surface potential variation with primary beam energy has traditionally been described as shown in Fig. 1 [6]. The surface potential has a positive value when the primary electron beam energy is between the "cross-over" 
points, $E_{1}$ and $E_{2}$. Under this condition more secondary and backscattered electrons are emitted from the surface than are injected by the primary beam. This charge imbalance produces a net positive voltage on the surface (normally $\leq$ $3.0 \mathrm{~V}$ ) that retains the lower energy secondary electrons until an equilibrium is established between incident and exiting electrons. Typical values for $E_{1}$ are around $100 \mathrm{eV}$ [7]. $E_{2}$ values have more variation, ranging between about 1.0 to $3.0 \mathrm{keV}$ depending upon the insulator [7]. For the positive surface potential condition, changes in the potential of conductors under the dielectric will polarize the insulator, producing a bound charge at the insulator's surface. The bound charge is temporary and the surface returns to the equilibrium conditions by retaining or emitting additional secondary electrons. The transient in secondary electron emission is observed as capacitive coupling voltage contrast (CCVC). While the time required to reach the positive surface equilibrium potential is inversely proportional to the incident electron flux, the value of the equilibrium potential has traditionally been thought to be dependent on the primary electron beam energy and independent of the beam current.

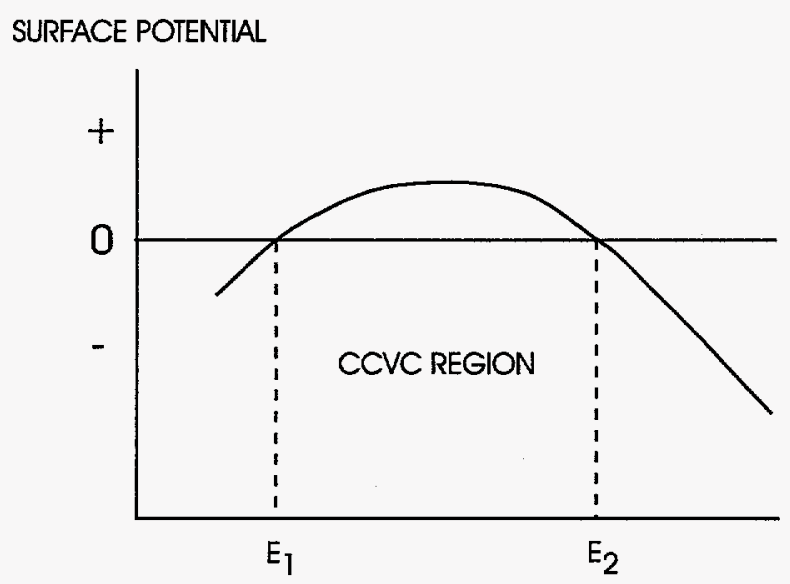

ELECTRON BEAM ENERGY

Fig. 1. Equilibrium surface potential of an insulator as a function of primary electron beam energy for low incident electron flux densities [6]. Energies between $E_{1}$ and $E_{2}$ are used for CCVC imaging.

Recent experimental work at Sandia indicates that, at high beam currents $(>100 \mathrm{nA})$, the surface potential is negative at beam energies between $E_{1}$ and $E_{2}$ [4]. We believe this occurs because the ability of the surface to produce secondary electrons is "saturated", as more electrons are being injected than can escape from the surface. A $1.0 \mathrm{keV}$ primary beam penetrates only about $0.05 \mu \mathrm{m}$, yielding a small volume for potential emission of secondary electrons. This negative charging effect has probably not been observed before because such high currents and low beam energies generate images with poor spatial resolution compared to those using conventional SEM parameters.

The negative surface charging at high beam currents and low beam energies can be used to produce an ac potential on the surface. This ac potential is generated by scanning the electron beam. The current density distribution of the SEM's electron beam is Gaussian as shown in Fig. 2. In the center of the distribution, the surface will charge negatively by the mechanism described above. The tails of the distribution (outside the dashed lines) will produce the traditional positive surface charge. Note that this positive potential will be reached quickly because the current density is still relatively high. As the primary beam scans across a point, the surface goes through a positive-negative-positive transition. The magnitude of the ac potential depends on the beam current and scan rate, with higher currents and faster scan rates generating larger changes in surface potential with time $(\partial V / \partial t)$.

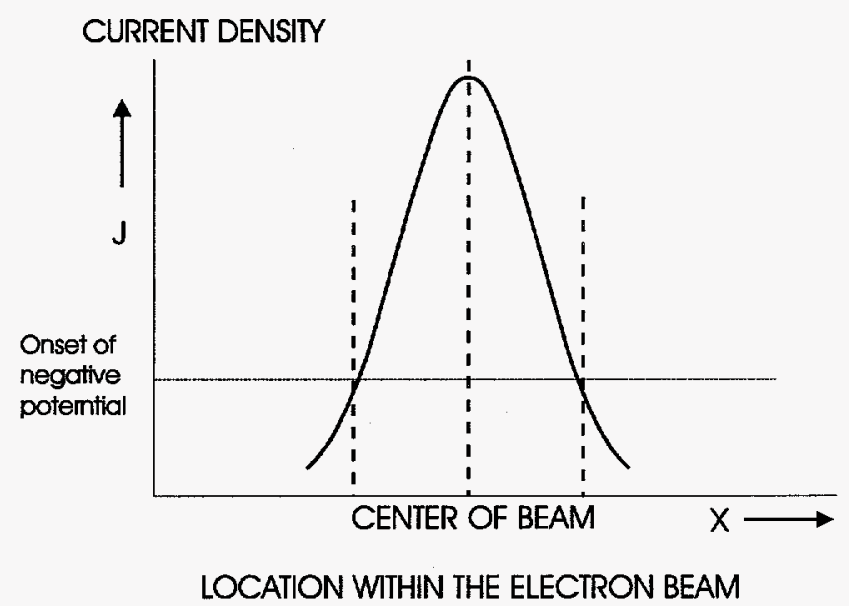

Fig. 2. Current density distribution of an SEM electron beam at the sample surface.

When the surfaces above passivated conductors are scanned with such an electron beam, the ac potential at the passivation surface will polarize the dielectric and produce a bound charge at the dielectric/conductor interface. This Capacitive Charge Generation (CCG) is similar to CCVC, but the dynamic charge and polarization originates from the passivation surface and not the buried conductor (see Fig. 3.). Increased scan rates will produce a larger CCG signal.

The ac bound charge from CCG has already been used to generate LECIVA images of open conductors [4]. LECIVA is similar to CIVA [3], but in LECIVA the potential of an electrically floating conductor is altered by the CCG bound charge instead of CIVA's direct electron injection. Faster scan rates produce a larger capcitively generated bound charge and hence greater LECIVA contrast, but the bandwidth response of the entire system must be considered to produce an optimum image. Fig. 4 is a secondary electron/LECIVA combined image example acquired using a 12 second per frame scan rate, a $200 \mathrm{nA}$ electron beam current, and a $1.0 \mathrm{keV}$ electron beam. The LECIVA signal is from an open metal-1 to metal-2 via on this 3 level metal Intel $386^{\mathrm{TM}} \mathrm{EX}$ microprocessor $[8,9]$. The open conductor is covered by a $10 \mu \mathrm{m}$ polyimide coating and approximately $3 \mu \mathrm{m}$ of nitride passivation. 


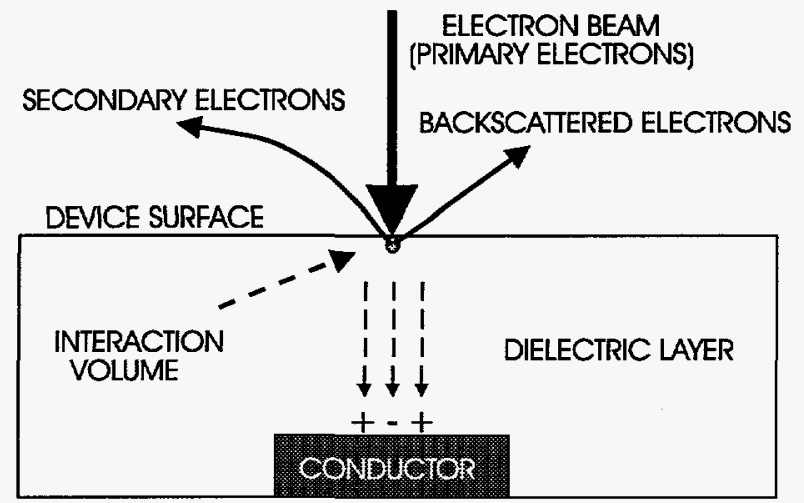

Fig. 3. The changing surface potential and induced bound charge produced by a high current, high scan rate electron beam incident on an insulator above a conductor.

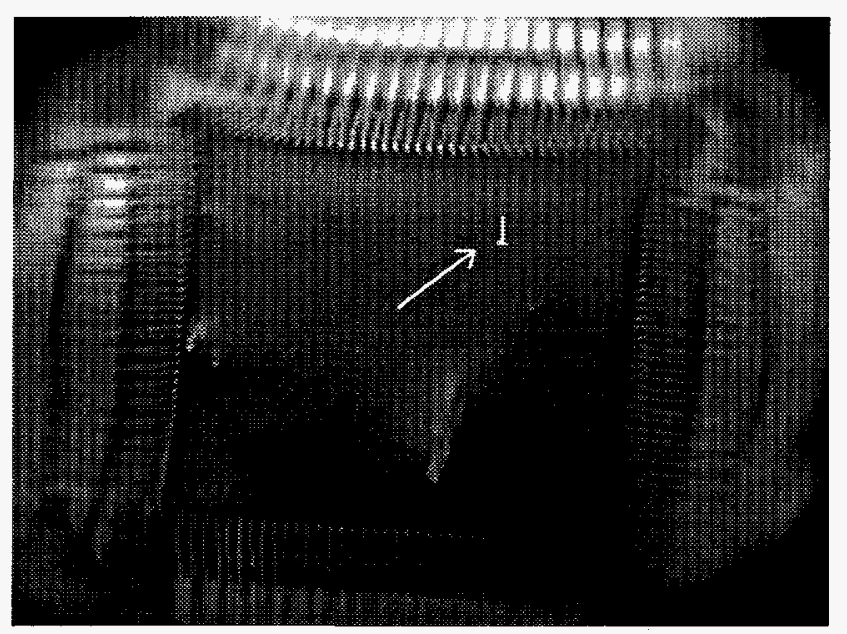

Fig. 4. Superposition of the LECIVA signal (white) with a secondary electron image of the same field of view. The LECIVA signal is indicated by the arrow.

The relatively large probing depth of the LECIVA example suggested that the CCG effect could be used to probe MCM interconnections with thick dielectric layers. However, instead of having a biased IC, MCM CCG analysis has been performed on unbiased interconnection networks by directly observing and imaging the current induced in MCM conductors.

\section{$C C G$ at Primary Electron Beam Energies above $E_{2}$}

CCG is also possible at primary electron beam energies above $E_{2}$ (Fig. 1). Above $E_{2}$ the primary electron beam penetration depth is deep enough that the scattered electrons at the bottom of the interaction volume do not have enough energy to reach the surface. This results in a net negative charging of the surface. As with positive surface charging, the literature does not address any variation of the surface potential with the beam scan rate or current other than that the surface will reach equilibrium more rapidly with increased beam current.

We have observed CCG at beam energies above the $E_{2}$ "cross-over" point. For the polymer passivations used in our examinations, this energy occurs between 1.0 and 3.0 $\mathrm{keV}$ (a maximum penetration of about $0.1 \mu \mathrm{m}$ ), but the effect has also been seen at higher energies (CCG has been observed at $20 \mathrm{keV}$ ). As with CCG at low primary electron beam energies (between $E_{1}$ and $E_{2}$ ), high beam currents and rapid scan rates are necessary for CCG. The ac charge generation at the surface has a different mechanism, however. We believe that the surface has a finite response time for secondary electron emission and this response time makes CCG possible at energies above $E_{2}$. At beam energies above $E_{2}$, the insulator surface will charge negatively as predicted. If the beam current is large enough, the negative equilibrium potential will be reached very quickly. When the scan rate is rapid in addition to having a high beam current, the primary beam will inject charge into an area on the insulator and move away from that area while secondary electrons are still being generated. If the response time of the insulator surface is significant compared to the scan's dwell time at a given point, a relative difference in surface potential will exist between the beam center and beam tail, with the insulator surface in the tail region being positive relative to surface exposed to the beam center. The currents induced in buried conductors by CCG using low primary beam energies indicate a predominantly negative bound charge and hence a predominantly negative surface charge transition from the equilibrium condition. The polarity of the currents produced by CCG at energies above $E_{2}$ indicate a predominantly positive bound charge and hence a predominantly positive surface charge transition from the equilibrium position.

In general, $C C G$ currents induced by operation above $E_{2}$ are larger than those produced by lower beam energies. In practice, however, if an adequate CCG current is generated energies below $E_{2}$ are used to reduce carbon contamination buildup on the surface.

Even larger CCG induced currents (two orders of magnitude higher) were observed by changing the primary electron beam energy while the electron beam is scanned. The effect is similar whether the change is between two energies both above $E_{2}$ or between two energies with one above and one below $E_{2}$. The effect is not seen if both primary beam energies are below $E_{2}$. The ac charging of the surface when the primary beam energy is increased produces a larger positive charge at the dielectric/conductor interface. This is consistent with an increased response time. The effect is transient, however; the increase in the current magnitude and the resulting image contrast decay in a fashion similar to a CCVC image. Decreasing the beam energy produces a transient current in the buried conductor in the opposite direction, also similar in appearance to a CCVC image. (Note that changing the beam energy of the SEM is very detrimental to the filament's lifetime, especially at high beam currents.)

\section{Optimizing CCG Probing Conditions}

CCG induces currents in conductors under dielectric layers that are too thick for direct electron beam interaction. 
Since the CCG signal generation process is dependent upon the capacitance between the electron beam probe (or the induced conductive layer formed by the probe) and the buried conductor, a model of this capacitance will provide an estimate of the signal magnitudes which can be generated for a given situation.

The simplest model for a capacitor is the parallel plate capacitor [10]. The ideal parallel plate capacitor ignores the contribution of fringing fields to the capacitance. This model, depicted in Fig. 5, is only accurate when the separation between the plates, $d$, is much smaller than the plate area A.

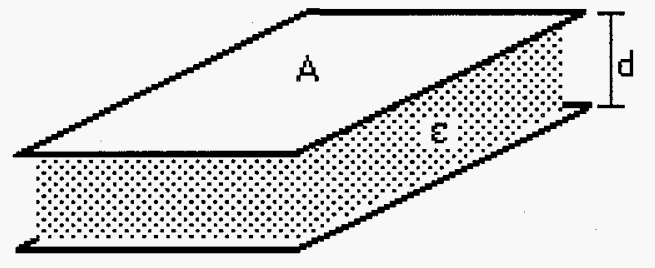

$$
C=\frac{\varepsilon \cdot A}{d}
$$

Fig. 5. Ideal parallel plate model for a capacitor where $\varepsilon$ is the dielectric constant.

In CCG, the situation is somewhat more complicated. The capacitance created by the induced conductive surface layer formed by the electron beam-sample interaction volume is most closely approximated by a concave, spherically shaped surface for the upper "plate" of the capacitor and a flat plate for the MCM interconnect as the bottom plate of the capacitor. As a first approximation, the capacitance for CCG is represented by the capacitance between a circular cylinder and a parallel plate. Using this model, the CCG capacitance is:

$$
\mathrm{C}_{\mathrm{CCG}} \approx \frac{\pi \cdot \mathrm{r}^{2}}{\mathrm{~d}}
$$

where $\mathrm{r}$ is the effective interaction volume diameter created by the electron beam. Using this model, the current collected by the CCG amplification system is given by

$$
\mathrm{i} \approx \mathrm{C}_{\mathrm{CCG}} \cdot \frac{\partial \mathrm{V}}{\partial \mathrm{t}}
$$

where the change in voltage with time is generated by both the scan rate of the primary electron beam and, if dynamic, the change in the primary electron beam energy.

The optimum CCG conditions will therefore depend on the sample being examined. If the dielectric is relatively thick and therefore the capacitance is small, a large $\partial \mathrm{V} / \partial \mathrm{t}$ is required to polarize the insulator and produce a CCG current. If the capacitance is larger (as in the case of thin dielectrics) a smaller $\partial \mathrm{V} / \partial \mathrm{t}$ is required. Slower scan rates are therefore possible on thinner dielectrics. The scan rate can also be kept at fast (TV) scan rates and a reduced amplification of the CCG induced currents used. This procedure permits real time examination with greater imaging bandwidth.

\section{CCG System at Sandia}

A block diagram of the CCG system at Sandia is shown in Fig. 6. A Cambridge $\mathbf{S} 200$ was used as an electron beam source. To maximize the beam current, the condenser lens

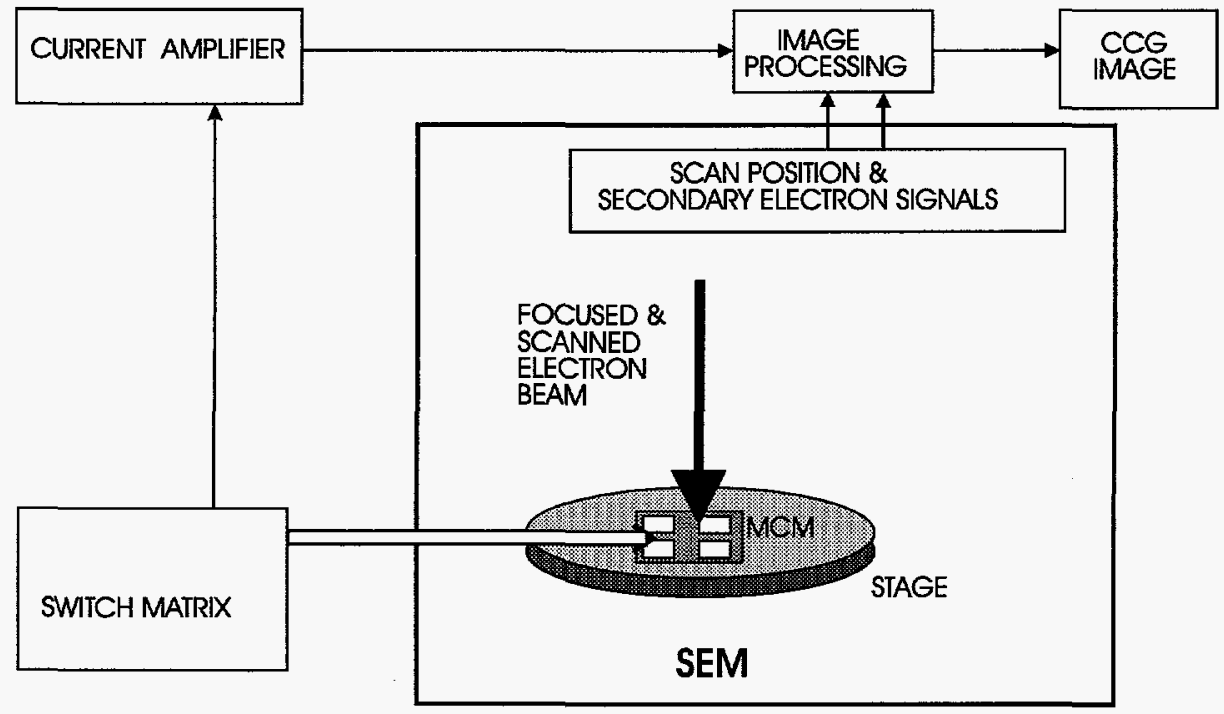

Fig. 6. CCG system. 
was set for maximum spot size and the final aperture was removed. A beam current of about $200 \mathrm{nA}$ is achievable under these conditions. The large beam yielded relatively poor spatial resolution for an SEM (approximately $0.5 \mu \mathrm{m}$ ) but was adequate for MCM interconnection analysis.

The MCM interconnections were examined as a passive conductor network with no applied bias. The currents induced in the conductors were measured and imaged using a GW Electronics Type 103 current amplifier at various gain settings. A switch box was used to select the conductor network of interest.

A fixture was constructed to maximize the working distance and therefore the field of view. The increased field of view facilitated observation of greater areas on the relatively large MCM structures. A working distance of 80 $\mathrm{mm}$ was obtained with the modified fixture, yielding an analysis area image diagonal of approximately $70 \mathrm{~mm}$ across the sample.

\section{CCG IMAGING OF MCM INTERCONNECTIONS}

The imaging examples described below demonstrate the probing capability of CCG on various MCM technologies. Two types of defects are of interest, short and open circuits embedded in the MCM interconnection. The examples show how the electrical continuity of a conductor can be viewed directly to determine its connectivity to other conductors in the sample. Different acquisition conditions are used to illustrate the tradeoffs in signal generation and image quality.

\section{Laminated Polymer Dielectric MCM}

Fig. 7 is a CCG image showing the continuity of a MCM conductor with sections covered by 30 and $60 \mu \mathrm{m}$ of polymer film dielectric. A cross-sectional schematic of the MCM interconnection layers is shown in Fig. 8. The top level, unpassivated conductor is used for bond pads. The image was acquired using a $0.3 \mathrm{keV}$ primary electron beam energy (below $E_{2}$ ), a $10^{9}$ gain on the current amplifier, and a 70 seconds per frame scan rate. The primary beam current for this and all the CCG images shown is approximately 200 $\mathrm{nA}$. The primary electron beam penetrates about $6 \mathrm{~nm}$ into the surface under these conditions. Fig. 9 is a CCG image taken under similar conditions with the scan rate increased to 12 seconds per frame. Notice the increase in signal produced by increasing the scan rate. The effective electron beam scanning speed can also be increased by decreasing the magnification. This effect is seen in Fig. 10 in which the magnification has been lowered and two additional MCM conductor paths are connected to the current amplifier. The scan speed is 12 seconds per frame.

Note that in all of the examples the bond wires and pads produce very strong contrast. The electrons from the beam are directly injected into the conductor at these sites and the currents produced are larger than the CCG induced currents.

The increase in CCG induced current that occurs with changing between two beam energies above $E_{2}$ during image scanning is shown in Fig. $11 . E_{2}$ is about $1.0 \mathrm{keV}$ for this polymer dielectric. The image shows the current produced immediately after a 1.6 to $1.7 \mathrm{keV}$ energy transition. The maximum beam penetration depth is about $0.1 \mu \mathrm{m}$. The image was acquired at a TV scan rate $(30$ frames per second) with a $10^{7}$ amplifier gain. The increased scan rate and changing beam energy produced an induced current two orders of magnitude greater than that in Fig. 10. Note that the polarity of the current (bright contrast) is opposite to that produced with beam energies below $E_{2}$ (dark contrast).

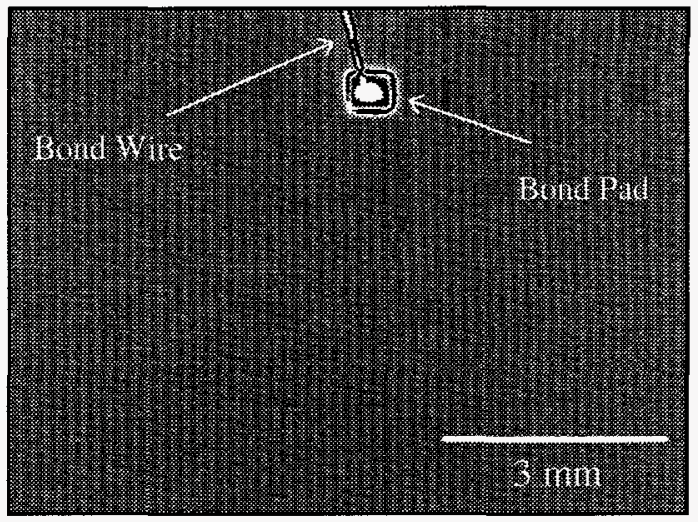

Fig. 7. CCG induced current image using a 70 seconds per frame scan rate.

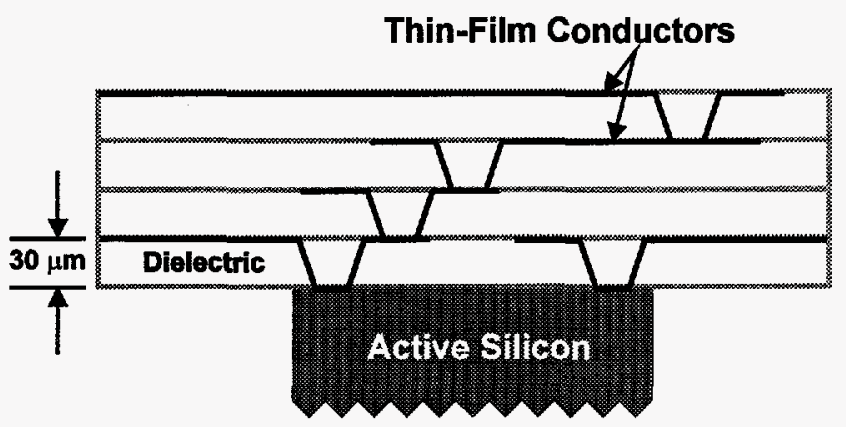

Fig. 8. Cross-sectional schematic of the technology examined in Fig. 7.

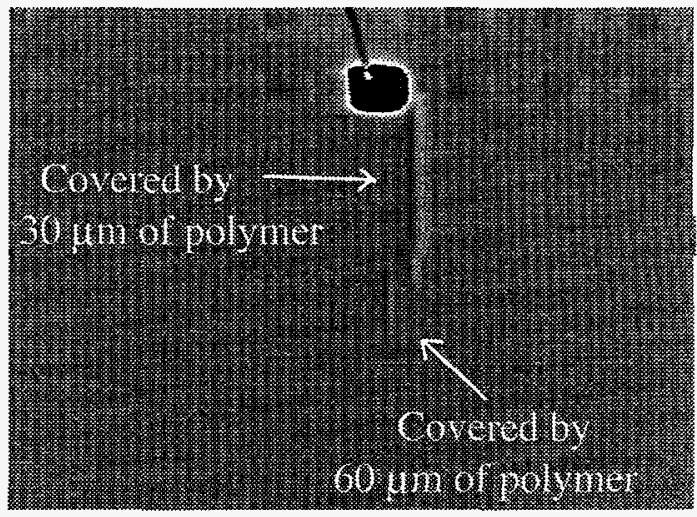

Fig. 9. CCG image of the conductor examined in Fig 8 using a 12 seconds per frame scan rate. 


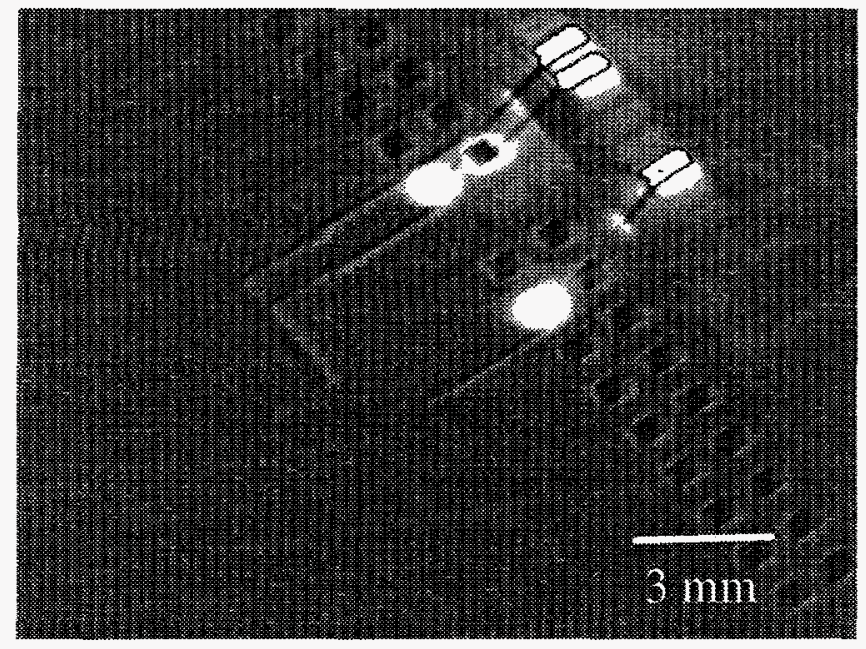

Fig. 10. Low magnification CCG induced current image displaying the continuity paths of $3 \mathrm{MCM}$ interconnections. A 12 seconds per frame scan rate was used.

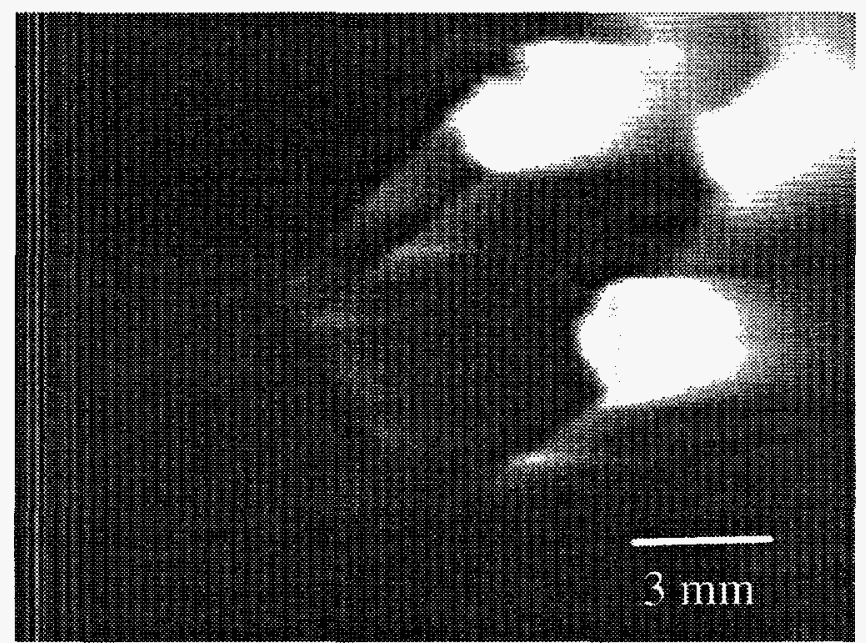

Fig. 11. CCG induced current image acquired at TV scan rates immediately after a primary beam energy change of 1.6 to $1.7 \mathrm{keV}$. The MCM interconnections are the same ones shown in Fig. 10.

The increase in CCG induced current by changing the beam energy above $E_{2}$ can also be seen at slower scan rates. Fig. 12a is a CCG image of a conductor under $30 \mu \mathrm{m}$ and 60 $\mu \mathrm{m}$ of polymer film as shown in Fig. 12b. The image was acquired immediately after a 1.6 to $1.7 \mathrm{keV}$ beam energy transition with a 12 seconds per frame scan rate. The amplifier gain was $10^{9}$. Fig. 13 was acquired under similar conditions to Fig. 12, but after the surface had been scanned for 1 minute. Note the reduction in image contrast.
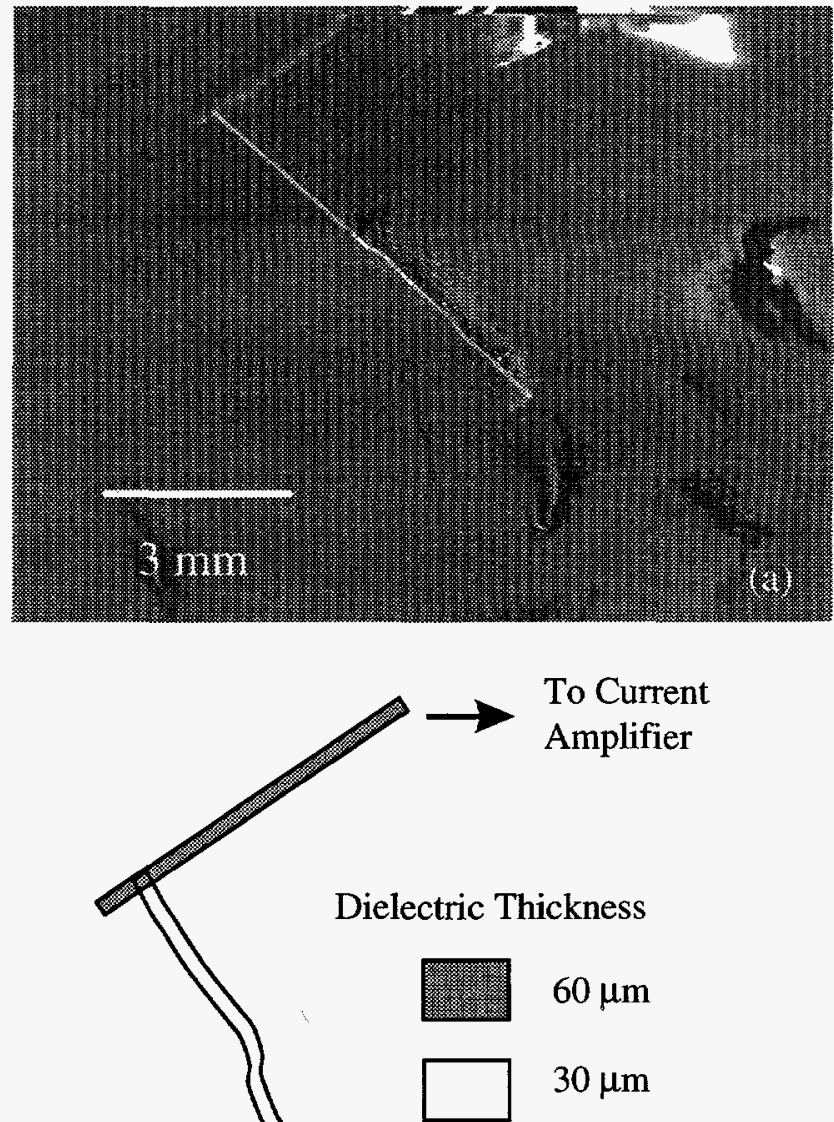

(b)

Fig. 12. (a) CCG induced current image taken immediately after a beam energy change from 1.6 to $1.7 \mathrm{keV}$. A 12 seconds per frame scan rate was used. (b) Planar layout of the two interconnection levels.

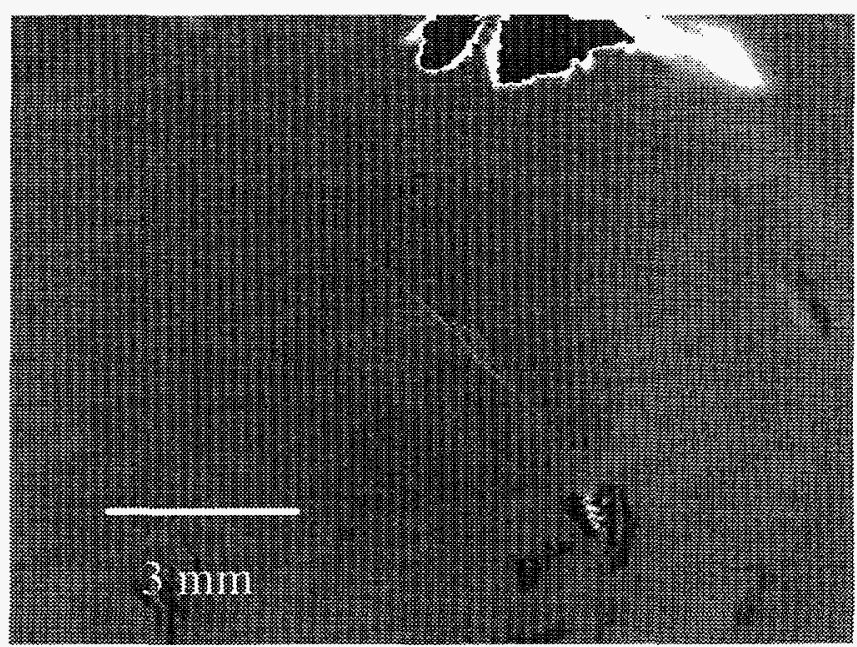

Fig. 13. Similar to Fig. 12, but acquired 1 minute of scanning at a beam energy of $1.7 \mathrm{keV}$ and a scan rate of 12 seconds per frame. 
Fig. 14 is a CCG example of an MCM interconnection approach that uses standard IC technology. Similar technologies are described in the literature [11]. This technology uses $1 \mu \mathrm{m}$ layers of $\mathrm{SiO}_{2}$ as an insulating dielectric. A cross sectional schematic of the interconnection layers is shown in Fig. 15. Fig. 14 was acquired using a TV scan rate at $1.0 \mathrm{keV}$ (below $\mathrm{E}_{2}$ ), with a $10^{7}$ amplifier gain. The large capacitance of the relatively thin (for MCM technologies) dielectric layers provides adequate contrast on the layers covered by 2 and $3 \mu \mathrm{m}$ of $\mathrm{SiO}_{2}$. Fig. 16 is a combined $\mathrm{CCG}$ induced current and secondary electron image which can be used for localization of the CCG signal.

\section{Flexible Circuit Cable}

Fig. 17a is a CCG example of a multilevel cable used in a Rigid/Flex printed circuit boards. This is not an MCM, but the insulating dielectrics are similar. Fig. $17 \mathrm{~b}$ is a secondary electron image of the same field of view. The conductors on the left and right are $50 \mu \mathrm{m}$ and $125 \mu \mathrm{m}$ below the surface respectively, as shown in the Fig. 18 schematic. The image was acquired below $E_{2}$ at $0.3 \mathrm{keV}$ with an amplifier gain of $10^{8}$. A TV scan rate was used. Note that the conductor on the left, inside the rigid printed circuit board, produces a weak contrast signal.

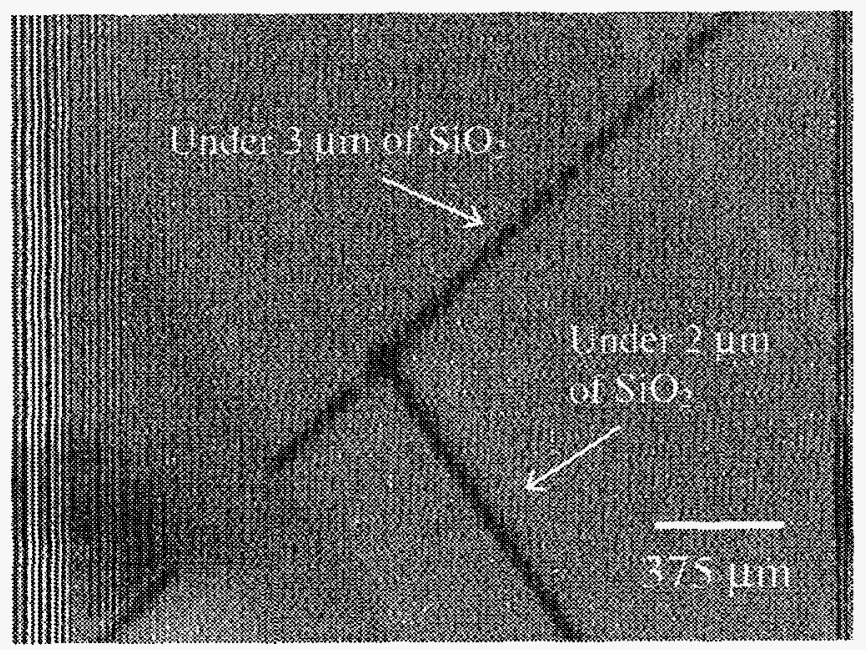

Fig. 14. CCG induced current image example of MCM interconnections under 2 and $3 \mu \mathrm{m}$ of $\mathrm{SiO}_{2}$.

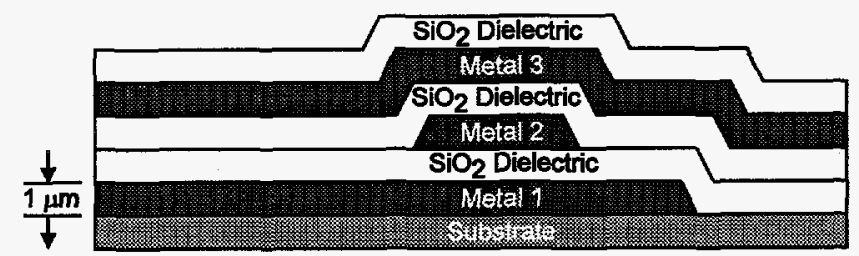

Fig. 15. Cross-sectional schematic of the technology examined in Fig. 14.

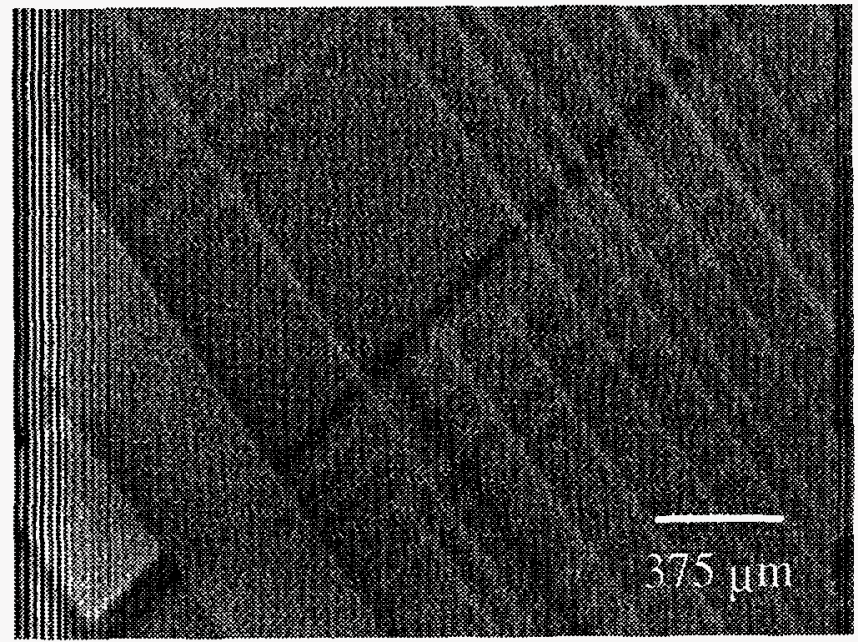

Fig. 16. Combined CCG induced current and secondary electron image.
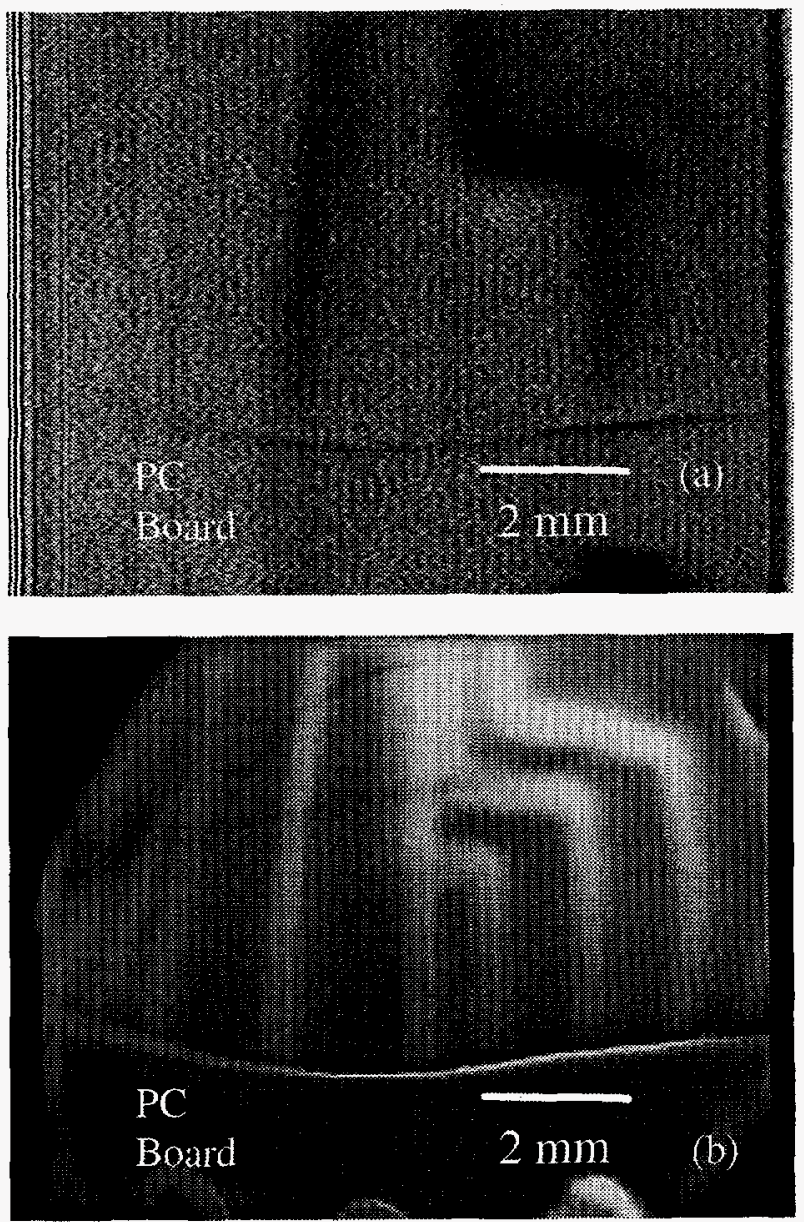

Fig. 17. (a) CCG induced current image of two conductors in a flexible circuit cable. (b) Secondary electron image of the same field view as Fig. 17a. The distortion in the images is an artifact of the low magnification. 


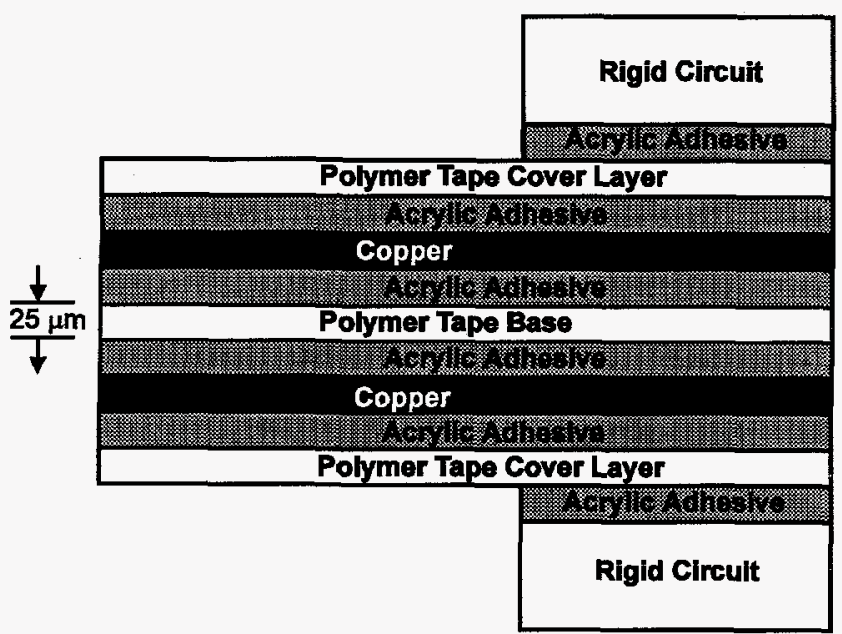

Fig. 18. Cross-sectional schematic of the flexible cable examined in Fig. 17.

\section{Embedded Polymer Dielectric MCM}

Fig. 19a is a CCG image of a solder bump MCM technology using a spun, cured polymer as an interlevel dielectric. A cross sectional schematic is shown in Fig. 20. Fig. $19 \mathrm{~b}$ was acquired at a TV scan rate, $0.5 \mathrm{keV}$ primary electron beam energy (below $E_{2}, 15 \mathrm{~nm}$ beam penetration), and an amplifier gain of $10^{8}$. The contrast is weak, but the conductor sections under 25 and $50 \mu \mathrm{m}$ of dielectric are visible. Fig. 19b is a TV rate secondary electron image of the same field of view taken at $0.5 \mathrm{keV}$.
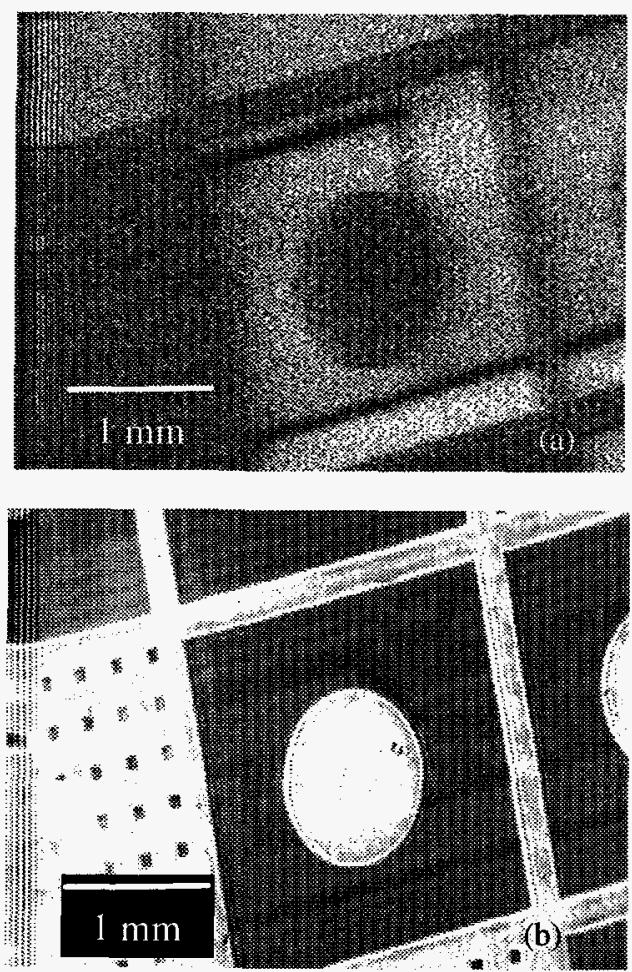

Fig. 19. (a) CCG image acquired at $0.5 \mathrm{keV}$ through 25 and $50 \mu \mathrm{m}$ of spun, cured polymer. (b) secondary electron image of the field of view shown in Fig. 19a.
Thin-Film Conductors

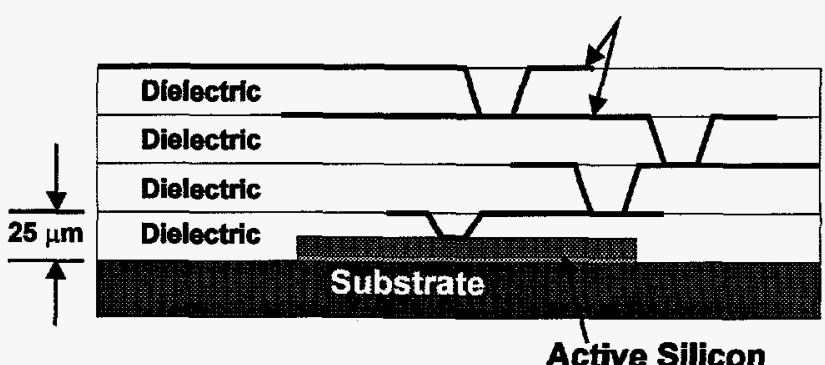

Fig. 20. Cross-sectional schematic of the technology examined in Fig. 19.

Fig. 21 is a CCG image of the same area as Fig. 19a, but the beam energy has been increased to $2.0 \mathrm{keV}$ (above E2, with about $140 \mathrm{~nm}$ beam penetration) and the amplifier gain reduced to $10^{7}$. Note the increase in signal and the polarity change compared to Fig. 19a. At scan rates of 1 second per frame and below no CCG induced currents were observed.

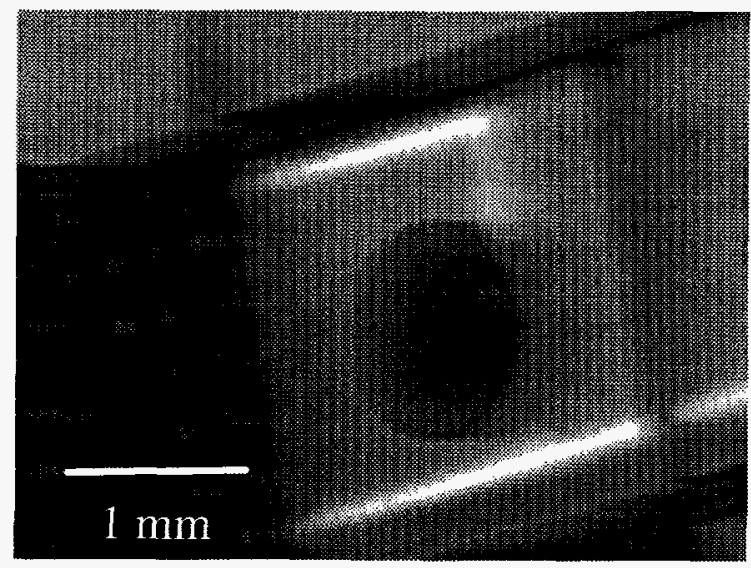

Fig. 21. Similar to Fig. 19 a, but a $2.0 \mathrm{keV}$ primary beam was used.

Fig. 22a demonstrates how CCG imaging can be used on the spun, cured polymer dielectric MCMs to locate the site of an open MCM interconnection. The open interconnection is covered by $25 \mu \mathrm{m}$ of polyimide. Fig. 22a was acquired using a $4.0 \mathrm{keV}$ primary electron beam $(0.4 \mu \mathrm{m}$ beam penetration), a TV scan rate, and a $10^{7}$ amplifier gain. This defective region had a hydrocarbon layer on the surface resulting from extensive examination using a $20 \mathrm{keV}$ primary beam. The surface carbon contamination altered the secondary electron emission characteristics so that a 4.0 $\mathrm{keV}$ beam was required for adequate CCG imaging. The interconnection is open under the metal mesh at the site indicated. By connecting the current amplifier to the other end of the conductor the open site can be confirmed as shown in Fig. 22b.

\section{Repatterened Die Micro Ball Grid Array}

A final example of CCG induced current imaging on MCM materials is given in Fig. 23. A cross sectional 
schematic of this repatterened die technology is shown in Fig. 24. Polyimide is used as the interlevel dielectric. Electrical testing of the device shown in Fig 24 indicated an open circuit between the two solder bumps with bond wires. The two solder bumps should be electrically connected through interconnections to a metal serpentine structure on the IC die. The question was where is the open circuit in the conductor path. Fig. 23 was acquired at $0.5 \mathrm{keV}$ ( below $\mathrm{E}_{2}$ with about $15 \mathrm{~nm}$ beam penetration), a TV scan rate, and $10^{8}$ amplifier gain. The continuity of the repatterning interconnections under $3 \mu \mathrm{m}$ of polyimide and on the serpentine structure under $6 \mu \mathrm{m}$ of polyimide is easily seen. The abrupt contrast change indicated by the arrow occurs under the solder bump and cannot be localized further, however the open was localized to the die and not the repatterning interconnections. Fig. 25 is an optical image of the micro ball grid array showing the interconnections and serpentine structure. The shading in the upper right is due to residual flux contamination during sample preparation.
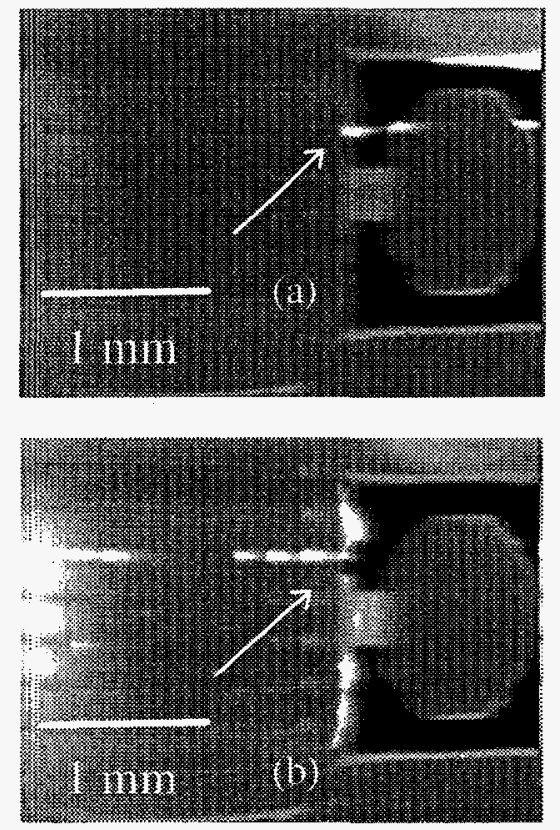

Fig. 22. (a) CCG induced current image acquired at 4.0 $\mathrm{keV}$. The arrow indicates the site of an open interconnection. (b) Similar to Fig. 22a, but the other end of the open interconnection is connected to the current amplifier.

\section{CONCLUSIONS}

The CCG techniques described for induced current generation provide new, non-destructive MCM probing capabilities through dielectric layers previously thought to be too thick for SEM analysis. They are powerful new additions to the set of MCM analysis tools for localizing open interconnections and verifying continuity. The techniques are also applicable to ICs with thick polymer passivations. Additionally, the new surface charging effects identified using high beam currents and rapid primary electron beam scan rates indicate a promising area for further surface physics analysis and additional technique development.

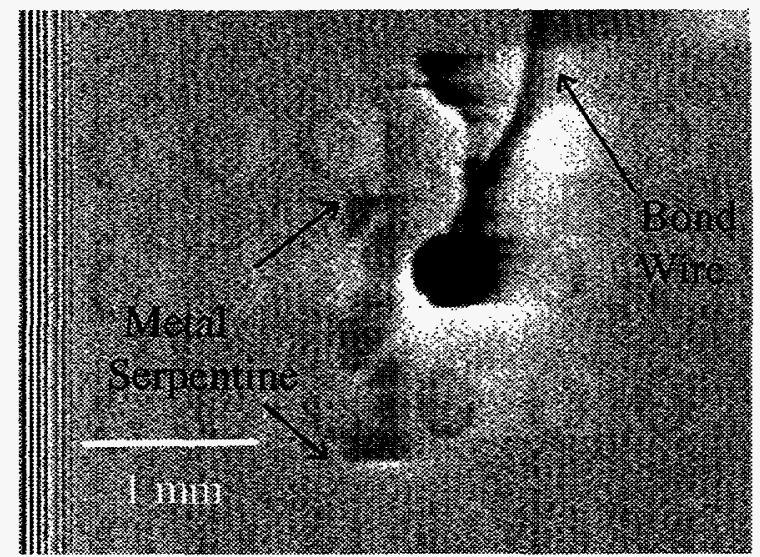

Fig. 23. CCG induced current image localizing an open interconnection to the die level.

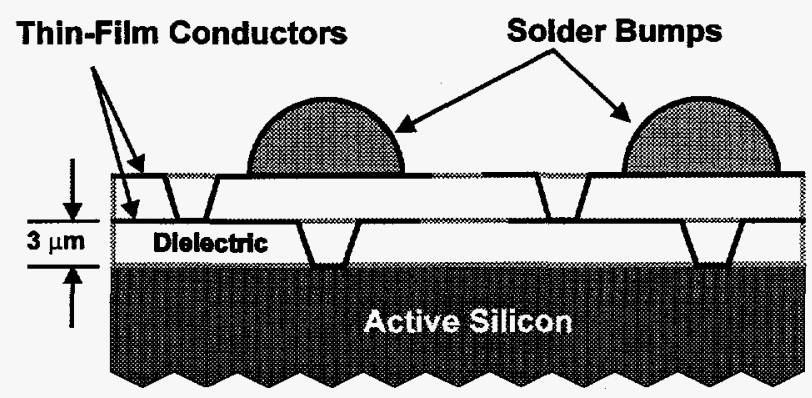

Fig. 24. Cross-sectional schematic of the technology examined in Fig. 23.

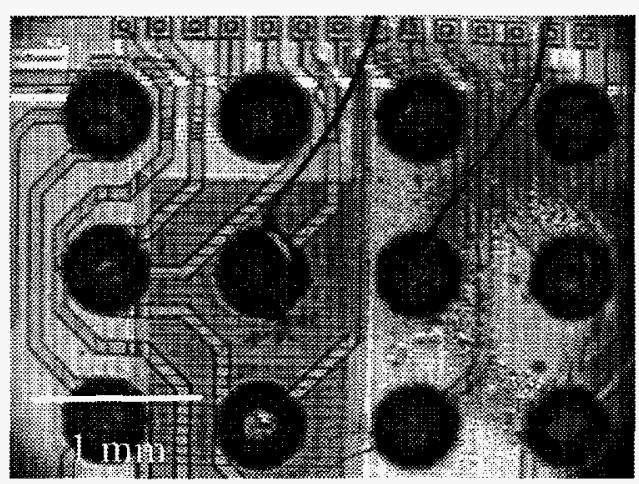

Fig. 25. Optical micrograph of the structure examined in Fig. 23.

\section{ACKNOWLEDGMENTS}

The authors thank Richard Anderson, Jerry Soden, and Paiboon Tangyunyong of Sandia National Laboratories for their careful review of the manuscript, Elaine Savage of ISA and Ron Anderson, Keith Treece, and Richard Gassman of Sandia for supplying MCM samples, and Kathy Myers of Sandia for sample preparation. The authors also thank Tony 
Miller of Intel Corporation for supplying samples for LECIVA analysis and permission to use Intel $386^{\mathrm{T} M \mathrm{EX}}$ failure analysis information. This work was supported by the U.S. Department of Energy under contract number DE-AC04-94AL85000.

\section{REFERENCES}

[1] Z. Sekulic, "The MCM Dilemma," Advanced Packaging, pp. 42-45,1992.

[2] C.A. Smith, C.R. Bagnell, F.A. DiBianca, E.I. Cole Jr., W.V. Oxford, and R.H. Propst, "Resistive Contrast Imaging: A New SEM Mode for Failure Analysis," IEEE Transactions on Electron Devices, ED-33, No. 2, pp. 282-285, 1986.

[3] E.I. Cole Jr. And R.E. Anderson, "Rapid Localization of IC Open Conductors Using Charge-Induced Voltage Alteration (CIVA)," Proceedings of the Int. Reliability Physics Symp., pp. 288-298, 1992.

[4] E.I. Cole Jr., J.M. Soden, B.A. Dodd, and C.L. Henderson., "Low Electron Beam Energy CIVA Analysis of Passivated ICs," Proc. of Int. Symp. for Testing and Failure Analysis, pp. 23-32, 1994.
[5] J. I. Goldstein, A. D. Romig, Jr., D. E. Newberry, C. E. Lyman, P. Echlin, C. Fiori, D. C. Joy, and E. Lifshin, Scanning Electron Microscopy and X-Ray Microanalysis, Plenum Press, 1992, pp. 106-111

[6] L. Reimer, Image Formation in Low-Voltage Scanning Electron Microscopy", SPIE Optical Engineering Press, Bellingham, WA, 1993, pp..71-73.

[7] K. Kanaya and S. Ono, Electron Beam Interactions with Solids for Microscopy, Microanalysis, and Microlithography", Scanning Electron Microscopy, Inc., AMF O'Hare, IL, 1984, pp. 69-98.

[8] H. Ahuja, D. Arriens, B. Schneller, V. Verma, and W. Whitman, "Intel386 ${ }^{\mathrm{TM}} \mathrm{EX}$ Embedded Processor IDDQ Testing," Proc. Int. Test Conf., 1995, pp. 902-909.

[9] T. Miller, J. Soden, and C. Hawkins, "Diagnosis, Analysis, and Comparison of $80386 \mathrm{EX} I_{D D Q}$ and Functional Test Failures," IEEE Int. Workshop on $I_{D D Q}$ Testing, 1995, pp. 66-68.

[10] D. E. Gray (editor), "American Institute of Physics Handbook," McGraw-Hill, 1972, chapter 5, pp. 12-19.

[11] P. Lall and S. Bhagath, "An Overview of Multichip Modules," Solid State Tech., Sept. 1993, pp. 65-76.

\title{
DISCLAIMER
}

\begin{abstract}
This report was prepared as an account of work sponsored by an agency of the United States Government. Neither the United States Government nor any agency thereof, nor any of their employees, makes any warranty, express or implied, or assumes any legal liability or responsibility for the accuracy, completeness, or usefulness of any information, apparatus, product, or process disclosed, or represents that its use would not infringe privately owned rights. Reference herein to any specific commercial product; process, or service by trade name, trademark, manufacturer, or otherwise does not necessarily constitute or imply its endorsement, recommendation, or favoring by the United States Government or any agency thereof. The views and opinions of authors expressed berein do not necessarily state or reflect those of the United States Government or any agency thereof.
\end{abstract}

